\title{
Coherent X-ray Scattering for Microanalysis at Taiwan Photon Source
}

\author{
Jhih-Min Lin ${ }^{1}$, Chun-Yu Chen ${ }^{1}$, Yi-Wei Tsai ${ }^{1}$ and $\underline{\text { Yu-Shan Huang }}{ }^{1^{*}}$ \\ 1. National Synchrotron Radiation Research Center, Hsinchu, Taiwan \\ * Corresponding author, jade@nsrrc.org.tw
}

The $3 \mathrm{GeV}$ Taiwan Photon Source (TPS) with low emittance $1.6 \mathrm{~nm}$-rad, which provides the extremely brilliant and highly coherent X-ray beam, is open to general users with e-beam current of $400 \mathrm{~mA}$ at the National Synchrotron Radiation Research Centre, Taiwan. The access to coherent X-rays at TPS opens up the possibility of building coherent experiment techniques. Taking this opportunity, the coherent X-ray scattering (CXS) TPS 25A beamline provides researchers to perform experimental techniques of smallangle X-ray scattering (SAXS), X-ray photon correlation spectroscopy (XPCS) and coherent diffraction imaging (CDI) for microanalysis studies [1-4]. Using XPCS and CDI with high coherent X-rays, ones can study the dynamics of a multitude of collective processes (e.g. diffusion of colloids) and three dimensional microstructures of materials.

The CXS beamline equipped with an in-vacuum undulator provides coherent photon flux greater than $10^{10}$ photons per second at $6 \mathrm{keV}$. The period length of the undulator is $22 \mathrm{~mm}$. The maximum deflection parameter is 2.07 for the undulator gap of $5 \mathrm{~mm}$. The photon source of the CXS beamline provides X-rays with an energy range from $5 \mathrm{keV}$ to $20 \mathrm{keV}$, based on the $3^{\text {rd }}, 5^{\text {th }}, 7^{\text {th }}, 9^{\text {th }}$ and $11^{\text {th }}$ harmonics of the undulator. Figure 1 shows the spectrum of X-rays from the undulator. The X-ray source sizes are about $6 \mu \mathrm{m}$ and $163 \mu \mathrm{m}$ in vertical and horizontal directions, respectively. In order to preserve the coherence of the photon source, the CXS beamline provides the possibility of a windowless operation with a differential pumping system. The X-ray beam is monochromated by a double crystal monochromator with energy resolution 2 x $10^{-4}$ by using Si (111) crystals. The X-ray beam is focused down to its size of $2 \mu \mathrm{m}$ at sample position by a vertical focusing mirror in the vertical direction. In the horizontal direction, the scheme of two-step focusing is applied to focus X-ray beam. The first horizontal focusing mirror is used to focus X-ray beam to a secondary source of size $50 \mu \mathrm{m}$ in the horizontal direction. A high quality slit at the secondary source varies its horizontal size of 50 to $5 \mu \mathrm{m}$. The following second horizontal focusing mirror therefore focuses $\mathrm{X}$-ray beam down to size of 10 to $2 \mu \mathrm{m}$ at sample position. In a summary, the Kirkpatrick-Baez (KB) mirrors formed by the vertical focusing mirror and the second horizontal focusing mirror are used to focus the $\mathrm{X}$-rays down to $2 \times 2 \mu \mathrm{m}^{2}$ in the energy range from $5.6 \mathrm{keV}$ to $20 \mathrm{keV}$. The high harmonic rejection of X-rays has been maintained by using two trip coatings Si and Rh/Pt of each mirror.

State-of-the-art detectors of Eiger X 16M and 1M are equipped for data collection of 133/750/3000 Hz frame rates with region-of-interest technique. To prevent the air absorption and scattering noise, a vacuum pipe with a pressure of $10 \mathrm{mTorr}$ is used to host detectors. The sample-to-detector distance can be varied with a range of 2 - 12 meters. The scattering vector can be varying from 0.0005 to $0.9 \AA^{-1}$. The Experimental Physics and Industrial Control System (EPICS) is used for the beamline control system. The Generic Data Acquisition system developed at Diamond Light Source is used for the data collection. An on-site storage farm at the CXS beamline provides the capacity of $200 \mathrm{~TB}$ for the 30-day data storage. A remote data center hosts one-year data storage for users. High performance computing system is provided for data acquisition and analysis.

Combined the highly coherent X-rays and state-of-the-art detectors, the CXS beamline at TPS provides 
advanced microanalysis techniques for millisecond and nanometer scales using SAXS, XPCS and CDI. The CXS beamline has been under commissioning since September in 2016. After successful achievements of commissioning, the experimental techniques of XPCS with time resolution of 2 milliseconds, SAXS, and CDI with spatial resolution of 10 nanometers are provided to general users in 2017. Furthermore, the CXS beamline will also provide users ptychography and Bragg CDI for microstructure studies. Ptychography and Bragg CDI are under commissioning. A gold Siemens star was used for the commissioning of ptychography technique by using coherent X-ray beam of $5.2 \mathrm{keV} .10 \mathrm{x} 10$ images were collected for analysis and reconstruction. The Siemens star is successfully reconstructed although the resolution of the preliminary result isn't optimized yet. Figure 2 shows a preliminary commissioning result of ptychography technique.

References:

[1] O. G. Shpyrko, J. Synchrotron Rad. 21 (2014), 1057.

[2] J. Miao et al., IEEE J. Sel. Top. Quant. Electron. 18 (2012), 399.

[3] J. M. Rodenburg et al., Phys. Rev. Lett. 98 (2007), 034801.

[4] G. J. Williams et al, Phys. Rev. Lett. 90 (2003), 175501

[5] Use of the Taiwan Photon Source is supported by the Ministry of Science and Technology of Taiwan.

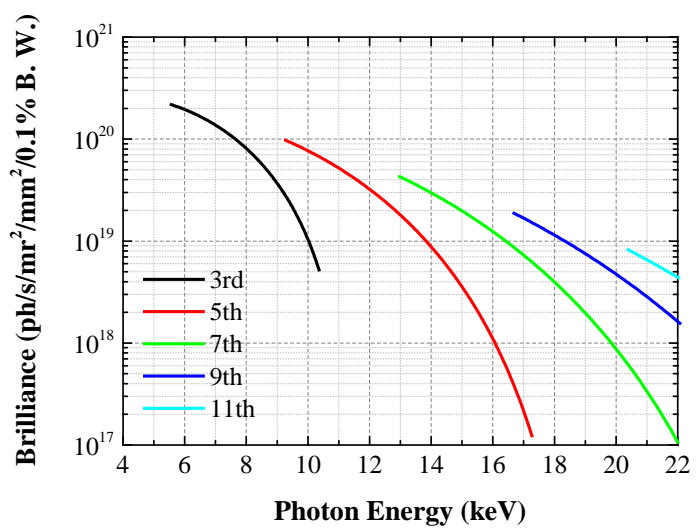

Figure 1. Brilliance of the $3^{\text {rd }}, 5^{\text {th }}, 7^{\text {th }}, 9^{\text {th }}$, and $11^{\text {th }}$ harmonics of the undulator of the CXS beamline.

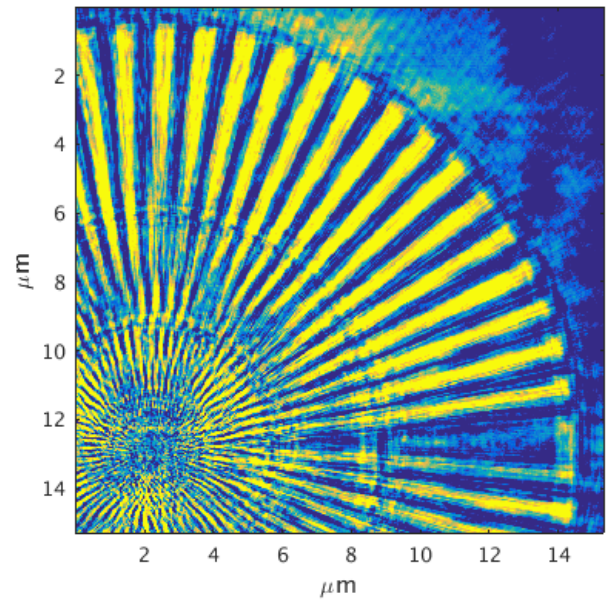

Figure 2. Preliminary reconstructed map of gold Siemens star by using ptychography. 